\title{
Optimizing RTS/CTS to Improve Throughput in Ad Hoc WLANs
}

\author{
Emilia Weyulu \\ Graduate department of Informatics \\ Tokyo University of Information Sciences \\ Chiba, Japan \\ Email: eweyulu@gmail.com
}

\begin{abstract}
IEEE 802.11 WLANs use carrier sense multiple access with collision avoidance (CSMA/CA) to initiate the Request to Send / Clear to Send (RTS/CTS) handshaking mechanism that solves the hidden node problem. However RTS/CTS also causes the exposed node problem where a node is unnecessarily prevented from accessing the wireless channel even when such access will not disrupt another nodes ongoing transmission. In this paper, we present continuing evaluation of a method for reducing exposed nodes in 802.11 ad hoc WLANs using asymmetric transmission ranges for RTS and CTS frames. NS2 simulations show that the proposed method improves overal network throughput in a topology scenario of a 3-D network faced with ceiling/floor obstructions.
\end{abstract}

\section{INTRODUCTION}

\footnotetext{
A LTHOUGH wireless local area networks (WLANs) provide mobility and convenience, their efficiency in today's high demand networks is unsatisfactory. WLANs generate a major portion of today's global Internet access due to their ease of use and their cost-effectiveness [1]. According to a Cisco report, wireless and mobile devices will generate $68 \%$ of all internet traffic by 2017 [2]. A factor driving the increase in use of wireless networks is the Internet of Things (IoT) currently in deployment. Devices such as household appliances, wearable devices and motor vehicles are being equipped with capabilities to connect to the Internet and to each other, wirelessly. In these scenarios, devices exist in close proximity to each other resulting in intense wireless channel contention which can lead to a severe degradation of the wireless network performance because of a high number of collisions.

The IEEE 802.11 MAC control is currently the most widely used medium access control protocol for WLANs [3]. In ad hoc networks, devices build automatic connections to other devices with no centralized infrastructure. The lack of centralized infrastructure to coordinate node activities gives ad hoc networks the advantage of simplicity but also makes them prone to collisions [3]. Since 802.11 networks do not detect collisions, frames suffering a collision will be lost in their entirety [4]. Thus, the goal in this type of networks is to avoid collisions whenever possible.
}

\author{
Masaki Hanada, Moo Wan Kim \\ Department of Informatics \\ Tokyo University of Information Sciences \\ Chiba, Japan \\ Email: \{mhanada, mwkim $\} @$ rsch.tuis.ac.jp
}

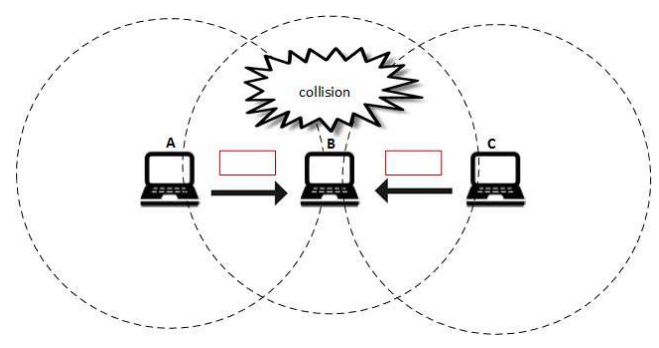

Fig. 1. The hidden node problem

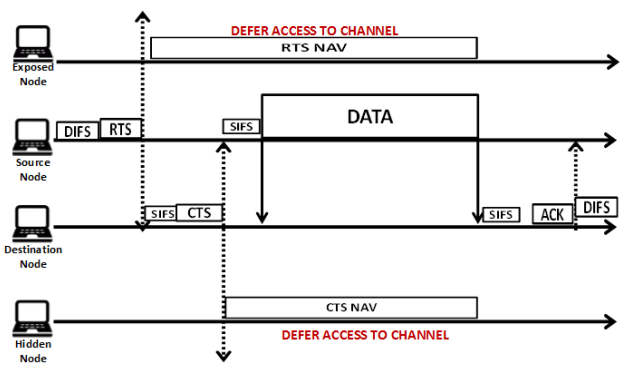

Fig. 2. The Standard RTS/CTS handshake

The hidden node problem occurs when a node is visible from one intermediate wireless node, but not from other nodes communicating with that node. In Fig. $1 B$ is the intermediate node. Both nodes $A$ and $C$ can communicate with node $B$, however, nodes $A$ and $C$ cannot sense each other since they are outside each others communication ranges and this leads to difficulties in the media access control layer. If node $A$ and node $C$ both start transmitting to node $B$ at the same time, packet collisions/loss occurs at node $B$.

\section{B. RTS/CTS handshake}

To solve the hidden node problem, the 802.11 MAC protocol includes an optional channel reservation scheme to help avoid collisions. This scheme is implemented through a CSMA/CA technique using the four-way RTS/CTS handshake shown in Fig. 2: In Fig. 2, a node with a packet to send, the Source Node, sends an RTS packet when it senses the

\section{A. Hidden node problem}




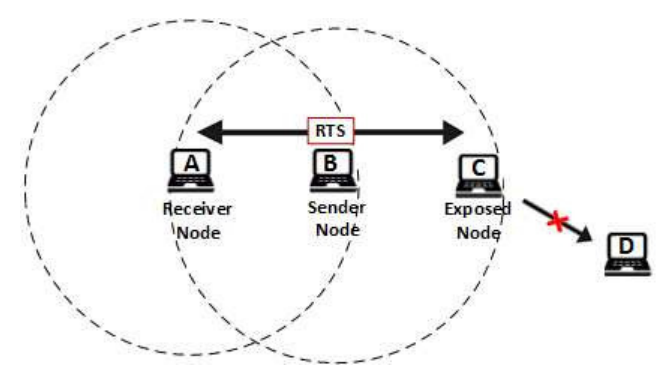

Fig. 3. The exposed node problem

channel being free for a period known as the DCF Interframe Space (DIFS). If the RTS packet is successfully received by the Destination Node without suffering collisions, it replies with a CTS after a period known as Short Interframe Space (SIFS). After receiving the CTS, the Source Node sends the data and waits for an acknowledgement (ACK) from the Destination Node to indicate successful transmission of the data i.e. DATA. Although the RTS/CTS exchange helps reduce collisions caused by hidden nodes, it also introduces another problem known as the exposed node problem.

\section{Exposed node problem}

Exposed nodes are nodes that are prevented from communicating with other nodes in their transmission ranges because they are close to a sending node and overhear the RTS frame [5]. Fig. 3 explains the exposed node problem. When node $B$ initiates a transmission to node $A$ by sending an RTS, node $C$ overhears the RTS and is forced to defer its planned transmission to another node, i.e node $D$. It is a mistake for node $C$ to not transmit to node $D$ just because it can overhear node $B$ 's transmission. Node $C$ 's transmission to node $D$ would not be a problem because it does not interfere with node $A$ 's ability to receive from node $B$. Thus, node $C$ is known as the exposed node in this scenario and has to hold its transmission for the Network Allocation Vector (NAV) period defined in the RTS frame. This decreases network spatial utilization and performance [6].

The CSMA/CA technique has not been improved since 1999 [7]. Although there have been several amendments to IEEE 802.11 standards since their ratification in 1997, CSMA/CA communication control technique has not caught up with the latest physical layer advancement. With the increased usage of wireless networks, it is important to optimize WLAN technology for the current and future environment.

In previous publications, we presented results for simulations based on an XY plane for grid and random distribution topologies [8]. In this paper, we introduce simulation results for an ad hoc WLAN deployed in an office building that represents an XYZ three-dimensional plane. This is in order to consider the elevation of the room and the attenuated received signal strength (RSS) in evaluating the difference in throughput between the standard RTS/CTS scheme and the proposed method.
The rest of this paper is organized as follows: Section II describes related works, Section III the proposed exposed node reduction idea and Section IV discusses the AODV routing protocol used to transport the data packet from sender to destination. Section V discusses the simulation set-up and results and Section VI concludes the paper.

\section{RELATED WORKS}

Our first research of the proposed method was reported in [6]. However, the research in [6] focused primarily on multi-rate transmission of RTS and CTS frames in order to control transmission range. Our method directly adjusts the transmission ranges of the control frames without considering transmission rate. This is done for simplicity and to not cause complications with the PHY layer convergence procedure (PLCP) preamble whose transmission rate cannot be changed. Additionally, the simulations in [6] were limited to evaluating the basic performance of the method with regards to only next-hop neighbour node communication. Our evaluations go a step further to evaluate end-to-end network communication by taking into account the routing protocol used.

Another method that effectively solves the hidden and the exposed node problems is the Dual Busy Tone Multiple Access (DBTMA) method described in [9]. DBTMA uses two out-of-band busy tones to protect the RTS packets and the DATA packets from interfering stations by assuming separate channels for tones and data. Although it is technically possible for wireless devices to communicate using multiple channels simultaneously, the MAC protocol in 802.11 networks is designed for a single channel only [10]. Hence, we only consider single channel communication in this research.

Other methods proposed in literature to solve the exposed node problem are such as that described in [11]. The method called Selective Disregard of NAVs (SDN) selectively ignores certain physical carrier sense and NAVs. This method needs additional functionalities to be implemented in nodes and lacks compatibility with the IEEE standard. Other MAC protocols based on Multiple Access with Collision Avoidance (MACA) were proposed in [12] that exploit control gaps between the RTS/CTS exchange and the subsequent DATA/ACK.

\section{PROPOSED IDEA}

\section{A. Overview}

The IEEE 802.11 standard performs RTS/CTS handshaking to avoid collisions by eliminating hidden nodes. However, the RTS/CTS scheme introduces another problem referred to as the exposed node problem from nodes close to a transmitting node that overhear the RTS frame.

\section{B. Asymmetric transmission ranges for RTS and CTS}

To mitigate the exposed node problem, the proposed method uses asymmetric transmission ranges for RTS and CTS frames. This is achieved by setting the transmission range of RTS frames to be less than that of CTS frames. We employ the fundamental method described in [6] to express the concept of asymmetric RTS/CTS transmission ranges as shown in Fig. 4. 


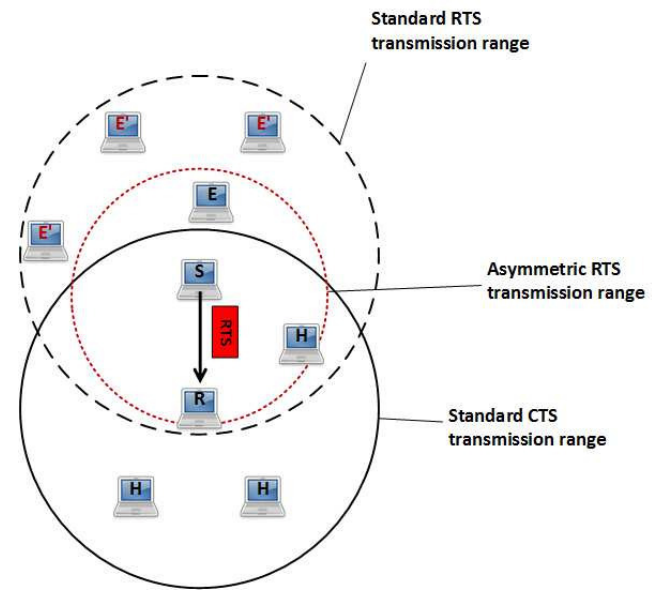

Fig. 4. Asymmetric RTS/CTS transmission

Reducing the transmission range of RTS frames means we eliminate some of the exposed nodes i.e. $E$ that were included in the original RTS transmission range; and overhear the RTS frame from the transmitting node, $S$. This means that the total number of exposed nodes in the network is reduced and can even be completely eliminated if the RTS range is included in CTS range. RTS frames do not need to have such a wide transmission range, as they only need to reach the receiving node in order to provoke a CTS response [6]. Thus if the transmission range of RTS is set to the minimum distance, only reaching the receiving node, this is enough to provoke CTS from the receiver node.

\section{AODV ROUTE DISCOVERY}

\section{A. Overview}

Nodes in an ad hoc WLAN cooperate in routing the data packets from the source node to the destination node since there is no centralized control. One of the most popular routing algorithms in ad hoc networks is the Ad Hoc On-Demand Distance Vector (AODV). AODV is a form of reactive routing that establishes routes between nodes only when they are requested and uses HELLO messages to discover neighbour nodes [13].

AODV uses hop count for choosing which route to use to transfer data from a source node to a destination node [13]. Because we are dealing with randomly placed nodes in our simulation, we need an efficient way to transmit the data packet from the source to the destination that incorporates the proposed asymmetric transmission ranges for RTS and CTS. In our work, we used the next-hop distance from AODV routing information to dynamically change the RTS transmission range.

\section{B. Received Signal Strength}

In multi-floor buildings, wireless signals can propagate through multiple floors in a phenomenon known as Inter-floor interference [14]. In such cases, a wireless node on Floor $-X$

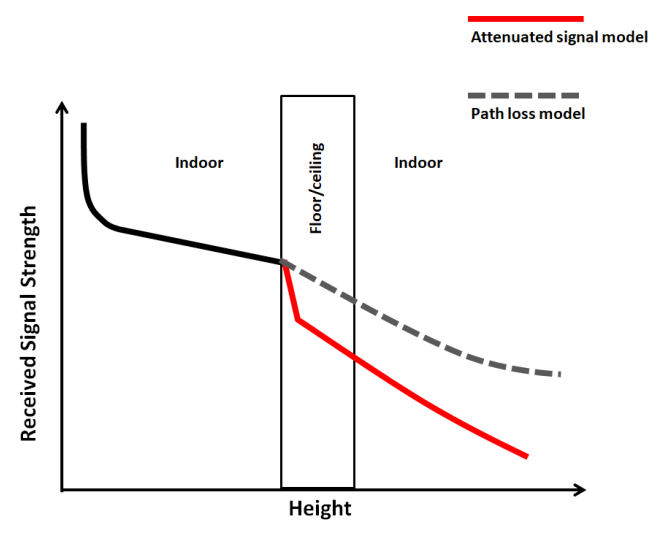

Fig. 5. Received signal attenuation model

will receive signals from nodes from the floors above and below, leading to interference issues.

In wireless networks, the received signal strength indicator (RSSI) can be used to estimate the distance between nodes [15]. Fig 5 shows a schematic of the received signal attenuation as it crosses the ceiling/floor. In the standard RTS/CTS method, the RSSI of RTS frames reaches adjacent floors, causing exposed nodes on those floors. RSSI decreases exponentially as the distance from the signal source increases [15]. In simulating the three-dimensional office-building scenario; we adjust the RTS transmission range considering the attenuation of the received RTS signal by the ceiling/floor. We use the RSSI attenuation model given in [15] using equations 1 and 2 below:

$$
\begin{aligned}
R S S I[d B m] & =-10 n \log _{10} \times \frac{d}{d 0} \\
d & =\frac{R S S I}{-10 n}
\end{aligned}
$$

In equations 1 and $2, n$ is the attenuation factor, $d$ is the distance from the node to the point of measurement and $d 0$ is a reference point distance. These parameters were used to calculate the indoor propagation environment of the RTS frames.

\section{NS-2 SIMULATION AND RESULTS}

\section{A. Overview}

We use the Network Simulator-2 (NS-2) to verify the proposed method. NS-2 is an open-source; event-driven simulator commonly used for communications research [16]. We use a simple power threshold scheme to control the transmission range of RTS frames. In 802.11, the transmission range of packet is determined by the power with which the packet is transmitted from the transmitting node. At the MAC level, we set a threshold that restricts how far an RTS frame goes as described in Algorithm 1. The newRTSThresh variable is compared to a received RTS packet's power level during runtime using Algorithm 1. If the power level is found to be larger than the level defined by newRTSThresh, the incoming RTS packet is simply discarded. In this way, we are 

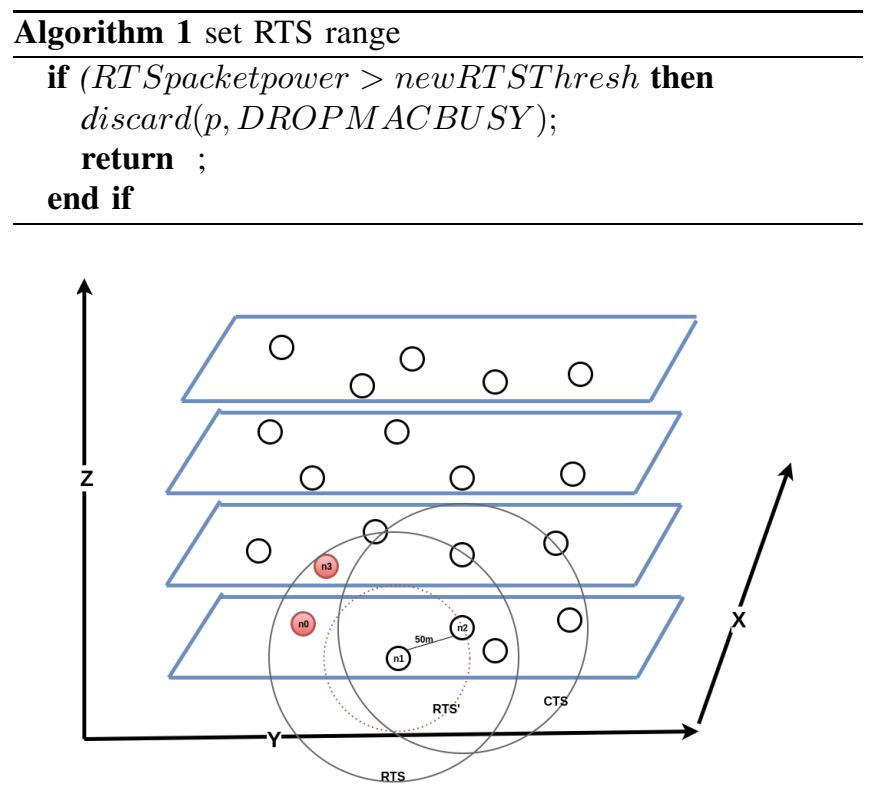

Fig. 6. The simulation model

able to confine RTS packet to a certain transmission range and in turn reduce the number of exposed nodes in the network.

\section{B. 3-D Simulations}

For the simulations, we assumed a small four-story office building to evaluate the asymmetric RTS/CTS method. The RTS transmission range was dynamically determined during runtime based on the next-hop distance as described in Section IV. We set the X-axis and Y-axis of the office floor to $180 \mathrm{~m}$ and the ceiling-to-floor height to $5 \mathrm{~m}$. The nodes on each floor were randomly distributed in such a way that each node was within $70 \mathrm{~m}$ of another node. This was to ensure that each pair of nodes were within the proposed method's RTS transmission range of half the CTS transmission range which was set to $140 \mathrm{~m}$.

In Fig. 6 which shows the simplified building model, the distance from $n 1$ to $n 2$ is $50 \mathrm{~m}$. Based on the information received from AODV's HELLO packets, the proposed method's RTS transmission range (i.e $R T S^{\prime}$ ) is set to $50 \mathrm{~m}$. From Fig. 6 , we can observe that $n 0$ and $n 3$ would have been exposed nodes using the standard RTS transmission range. and Table. I presents the rest of the simulation parameters and conditions:

\section{Analysis of results}

We present simulation result comparison between the Standard and the Asymmetric RTS/CTS methods. Fig. 7 shows packet drops between the two methods for the 25 nodes simulated in our 3-D scenario. The Standard method experiences a higher number of CBR packet drops per node with the overall number of drops for the network at 1400 packets in comparison to the Asymmetric method with an overall packet drop of 507. This means the proposed method provides a $63.8 \%$ reduction in packet drops. The packet drops from unsuccessful CBR packet transmissions in the Standard method

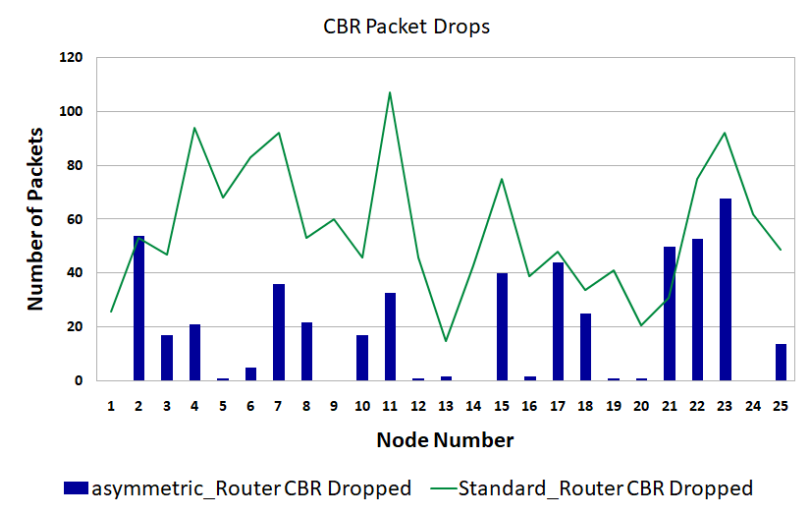

Fig. 7. Throughput results

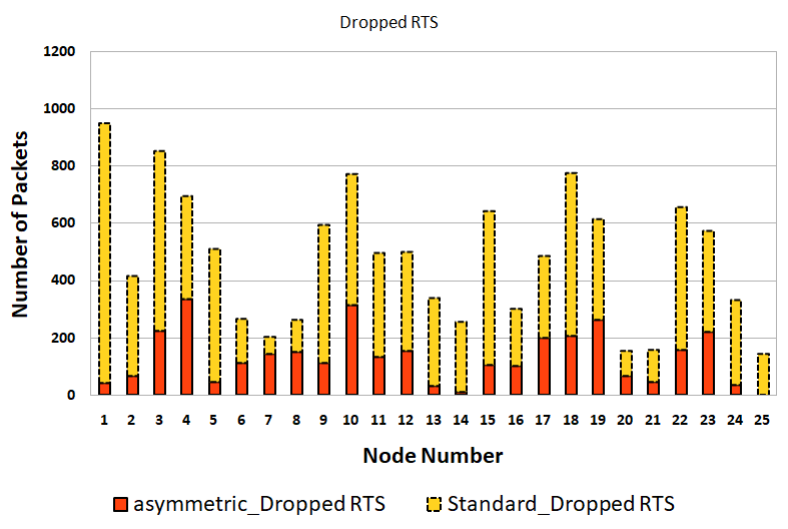

Fig. 8. Throughput results

are a result of secondary transmission failures as exposed nodes are prevented from communicating with other nodes in their communication ranges. Fig. 8 shows the number of dropped RTS packets between the two methods. The Standard method has an overall high number of dropped RTS packets than the Asymmetric method. RTS packet are dropped when the retry count set at the MAC layer is exceeded (after 7 failed RTS transmissions in NS-2). However; because some exposed nodes around the receiver have already received the failed RTS packet, they enter the NAV period and back-off from accessing the channel. With the Asymmetric method, even with RTS packet failures, some nodes around the sender will still be able to communicate leading to the increase in throughput presented in Fig. 9. Fig. 9 shows the throughput comparison between the Standard RTS/CTS and the proposed Asymmetric RTS/CTS methods for the 25 randomly distributed nodes using no RTS/CTS, using the standard RTS/CTS method and using the Asymmetric RTS/CTS method. Throughput was calculated using Equation 3:

$$
\text { Throughput }=\frac{\text { TotalReceivedPackets } \times \text { PacketSize }}{\text { RoundTripTime }}
$$

From Fig. 9, we can clearly see that the Asymmetric RTS/CTS 
TABLE I

SIMULATION PARAMETERS

\begin{tabular}{|l|l|l|}
\hline \multicolumn{3}{|c|}{ Transmission range } \\
\hline Frame Type & Standard RTS/CTS & Asymmetric RTS/CTS \\
\hline RTS & $140 \mathrm{~m}$ & Next-hop node distance \\
\hline CTS & $140 \mathrm{~m}$ & $140 \mathrm{~m}$ \\
\hline \multicolumn{3}{|c|}{ Other parameters } \\
\hline Data packet size & 3000 bytes & \\
\hline Propagation model & TwoRayGround & \\
\hline Routing protocol & AODV & \\
\hline \multicolumn{3}{|l|}{ Simulation conditions } \\
\hline Simulation time & 60 seconds & \\
\hline Simulation frequency & x300 & \\
\hline Communication start time & Uniform Random & \\
\hline
\end{tabular}

Throughput

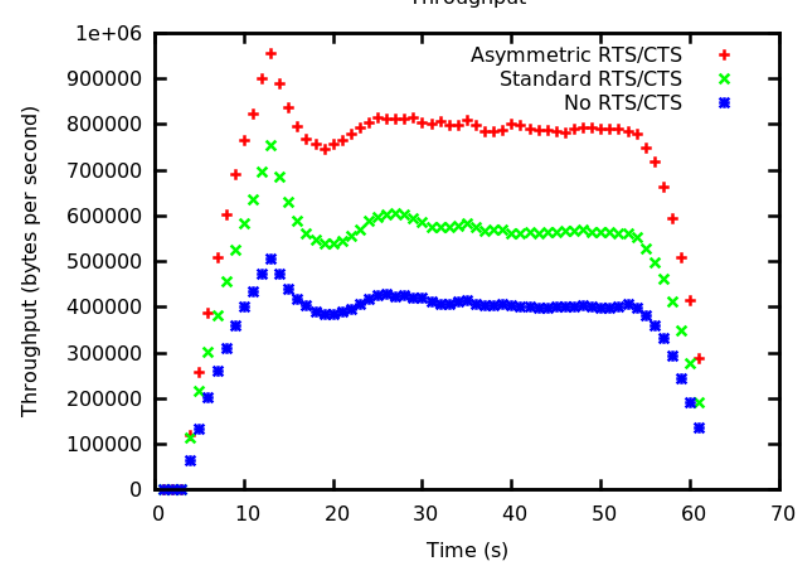

Fig. 9. Throughput results

method has significantly higher throughput even in scenarios with obstacles such as the ceiling or floor. We attribute this throughput gain to the elimination of exposed nodes in the network.

\section{CONCLUSION}

In this paper, we present further evaluation of a novel method for reducing exposed nodes in ad hoc WLANs using asymmetric transmission ranges for RTS and CTS frames. To set the RTS transmission range, we used the next-hop node selected by the AODV routing protocol to determine the best route to the destination while keeping the RTS range at a minimum. We employ an RSSI attenuation model to consider obstacles to the wireless signal in our simulated 3-D model. Simulation results show that the proposed method has better overall network throughput than the standard method.

Future work will look at the effect the proposed method has in scenarios of mobile nodes in indoor settings. Furthermore, we will study the impact of a destination having multiple sources. These evaluations will allow us to further validate the usefulness of the Asymmetric RTS/CTS idea and propose it as an adjustment to the RTS/CTS standard in IEEE 802.11 WLANs.

\section{ACKNOWLEDGMENT}

The authors would like to thank Dr Akihisa Matoba for his assistance with this research.

\section{REFERENCES}

[1] iPass Inc. press, "iPass WiFi growth map shows 1 public hotspot for every 20 people on earth by 2018 ," http://www.ipass.com/pressreleases/ ipass-wi-fi-growth-map-shows-one-public-hotspot-for-every-20-people -on-earth-by-2018/

[2] Cisco, "Visual Networking Index: Global Mobile Data Traffic Forecast Update, 20162021 White Paper," http://www.cisco.com/c/en/ us/solutions/collateral/service-provider/visual-networking-index-vni/ mobile-white-paper-c11-520862.html

[3] F. Gebali Analysis of Computer Networks, Springer International Publishers, 2015

[4] J. F. Kurose and K. W. Ross, Computer networking: a top-down approach, Boston: Pearson, 2013.

[5] K. Xu, M. Gerla and S. Bae, "Effectiveness of RTS/CTS handshake in IEEE 802.11 based ad hoc networks," Ad Hoc Networks, vol. 1(1), pp. $107-123,2003$.

[6] A. Matoba, M. Hanada, H. Kanemitsu, and M. W. Kim, "Asymmetric RTS/CTS for Exposed Node Reduction in IEEE 802.11 Ad Hoc Networks," JCSE, vol. 8, No. 2, pp. 107-118, 2013

[7] H. A. Omar, K. Abboud, N. Cheng, K. R. Malekshan, A. T. Gamage and W. Zhuang, "A Survey on High Efficiency Wireless Local Area Networks: Next Generation WiFi," IEEE Communications Surveys \& Tutorials, vol. 18(4), pp. 2315-2344, 2016.

[8] E. Weyulu, T. Iwabuchi, M. Takeshi, M. Hanada and M. W. Kim, "Ad hoc WLAN throughput improvement by reduction of RTS range," 2017 19th International Conference on Advanced Communication Technology (ICACT), Pyeongchang, Kwangwoon Do, South Korea, pp. 247-251, 2017.

[9] Z. J. Haas and J. Deng, "Dual busy tone multiple access (DBTMA)-a multiple access control scheme for ad hoc networks," Communications, IEEE Transactions on, vol. 50, pp. 975-985, 2002

[10] J. So and N. H. Vaidya, "Multi-channel mac for ad hoc networks," Proceedings of the 5th ACM international symposium on Mobile ad hoc networking and computing - MobiHoc '04, 2004.

[11] L. Jiang and S. C. Liew, "Improving Throughput and Fairness by Reducing Exposed and Hidden Nodes in 802.11 Networks," IEEE Transactions on Mobile Computing, vol. 7, No. 1, pp. 34-49, 2008

[12] P. Karn, "MACA A new channel access method for packet radio," in ARRL/CRRL Amateur Radio 9th Computer Networking Conference, pp. 134-140, 1990

[13] C. E. Perkins and E. M. Royer, "Ad-hoc On-Demand Distance Vector Routing," Proc. 2nd IEEE Wksp. Mobile Comp. Sys. and Apps., pp. 90-100, 1999

[14] CISCO, "Multi-Floor Deployments," http://www.cisco.com, 2014

[15] A. S. Kim, J. Hwang and J. Park, "Enhanced Indoor Positioning Algorithm Using WLAN RSSI Measurements Considering the Relative Position Information of AP Configuration," Journal of Institute of Control, Robotics and Systems, vol. 19, 146-151, 2013.

[16] T. Issariyakul and E. Hossain, Introduction to Network Simulator NS2, Boston, MA: Springer US, 2012 